\section{LA PERMÉABILITÉ DES FRONTIÈRES}

Jocelyne Alloucherie

RÉSUMÉ: Après avoir été invitée par Engramme, j’ai produit une gravure à partir du transfert d'une image photographique en me servant de la technique de la photogravure. Fondée sur ces différentes images, j’amorce une réflexion sur les différences entre des processus distincts en tenant compte de leurs qualités matérielles et esthétiques.

Mots-clés: processus créateur. dessin. photographie. gravure. image numérique.

La réflexion que je livre ici est issue d'une pratique; elle est donc fragmentaire et incomplète. II se peut aussi qu'elle vous semble dichotomique. Mon intention de départ était très simple; nommer des différences et des similitudes entre des images semblables reproduites par divers moyens. Pour de multiples raisons politiques et contextuelles, j’ai bifurqué quelque peu vers une interrogation rapide mais importante sur la nature fondamentale de l'image.

J'ai épinglé au mur quatre images légèrement différentes avec la volonté d'y laisser errer le regard et de déceler des indices pertinents; un geste spontané qui s'est vite révélé naif. La première de ces images montrait le dessin d'une ombre, la seconde la photographie d'une ombre; la troisième était une eau forte et la quatrième une impression au jet d'encre. Des ombres toujours; les traces aléatoires d'une pure graphie solaire. Elles étaient donc à leur origine déjà, des images, des ersatz; sur le mur, elles se déployaient côte à côte comme les figures d'une redondance conceptuelle; des images d'images, substituts de substituts. L'effet était agréable à l'œil et le tout paraissait toujours simple, ouvert à une large extrapolation; j’ai donc plongé, j’ai cherché, je me suis perdue dans un labyrinthe de considérations diverses; sur les rapports de l'image singulière à son médium, ceux de l'image reproduite à l'idée de multiple; les rapports

Texte initialement publié dans les actes du colloque L'Estampe Contemporaine. Québec: Engramme, 2006. de l'œurre unique à un médium mécanique de reproduction; jai erré dans des strates se recoupant, se contredisant, se renforçant, pour finalement me heurter à l'impossibilité de dégager des constantes. Si je serrais un aspect, il était suivi immédiatement d'un déni, surgissant de la mémoire d'une autre oeuvre et défaisant toute certitude. J'ai dû revenir à cette évidence; à ce fait que pourtant je connaissais bien; les rapports cherchés doivent êtres redéfinis à chaque œurre nouvelle. C'est bien le propre de l'art pense-t-on et il ne saurait y avoir d'autre loi que celle qui se précise dans l'œurre singulière et s'y épuise. Mais cette inconsistance, cette fluidité bien connue des choses artistiques comportait ici un plus; un insaississable, venant de la nature même de l'image; de la définition du concept d'image. J'ai utilisé ce terme d'image presque machinalement, afin de cerner une catégorie très générale. Et vous l'avez tous, sans doute, associé immédiatement et tout aussi machinalement à l'idée de figuration. Nous oublions que l'image est un concept antérieur à la figuration, beaucoup plus complexe. L'image procède directement de l'imaginaire, alors que la figuration est un duplicata plus ou moins fidèle du réel. Une figuration est toujours une manifestation relativement précise, l'image reste une potentialité; elle existe antérieurement à son incarnation; elle flotte dans la pensée, impalpable, en quête d'une forme. Nous disons souvent j'ai une image de quelque chose, il me vient une image de... Cette question, qu'est-ce que l'image, semble absurde mais se révèle tout aussi problématique que celle qui se pose devant une définition de la beauté. Bien que, de cette dernière, on puisse affirmer avec certitude qu'elle est de la nature de l'expérience; qu'elle se présente dans la richesse d'une expérience sensible. Le concept d'image serait à la fois plus précis et plus vaste. On parle de l'image parfois comme d'une vague idée. On sait qu'elle évoque, qu'elle représente; elle est un substitut pour quelque chose, une réalité mentale d'abord; la clef d'articulation de structures imaginaires complexes; une irréalité avant d'être matérialisée. Elle devient alors porteuse de références, mais elle n'est pas nécessairement une figuration. Une abstraction évoque aussi, représente et réfère; même si ce n'est qu'à un concept sans figure. Une abstraction peut encore devenir une image de sa propre histoire. Elle appartient alors aux reprises de styles, aux maniérismes, conscients ou non. Comme substitut, peu importe son degré de fidélité au réel, l'image nous inscrit dans une distance mentale. Et c'est ici que nous touchons à sa véritable nature. Elle nous déplace dans l'illusion d'une présence fictive; nous exclue d'une immédiateté temporelle. Toute image reste insaisissable, flottante, dans cette double mesure, cette nature contradictoire; elle nous implique et nous tient à l'écart; elle agit dans l'inclusion et l'exclusion. Elle 
est la dualité même. Certains moyens de la représenter accentuent cette particularité.

La photographie qui témoignerait au plus près de notre vie quotidienne et intime nous abstrait paradoxalement et totalement de cette réalité. Nous n'en sommes plus dupes; nous préférons en cultiver l'ignorance car le leurre d'une fausse inclusion nous berce confortablement. C'est comme si nous y étions. Une formule qui résume bien d'ailleurs tout le pouvoir de l'image médiatique construit sur la conscience de cette puissante illusion; plus le moyen de rendre l'image se fait fidèle à une certaine réalité, l'imite, plus cette dimension opère en force. Cette connaissance et cette utilisation de l'ambivalence ontologique de l'image maintiennent la toute-puissance des médias dans une culture de l'image Le pouvoir de ce qu'ils affirment est magnifié par la double structure de celle-ci; qui semble toujours nous inclure alors qu'elle nous tient irrémédiablement à distance dans une participation négative; en prenant en charge notre passivité consentante elle sauve aussi notre bonne conscience. Nous aurons l'impression d'avoir pris parti pour une cause. Et cette passivité ou l'image médiatique nous confine par l'illusion, s'accroît encore par l'usure, par la lassitude d'une diffusion répétée. Par la banalisation. Historiquement, le désir de transgresser la fourberie première de l'image s'est révélé aussi constant et insistant que celui de l'affirmer. Le culte de celle-ci a toujours été jumelé d'un désir d'iconoclastie. Même l'histoire de l'abstraction relève de cette tension. L'image, en Occident, a un lieu privilégié; le rectangle devant soi qui en a été défini comme le site idéel depuis des siècles. Lieu où elle se retrouve à la fois comme miroir, face-à-face, vis-à-vis; espace de la frontalité. l'écran contemporain a semblé apporter autre chose à la structure de l'image; un élément qui relève de cette ancienne fascination du théâtre où la conscience de l'illusoire est accentuée et concrètement marquée par la frontière que constitue la scène laissant ainsi agir l'illusoire dans toute sa force sans que soit jamais perdue la conscience de l'exclusion. Cela vaut pour le cinéma, mais la vidéo opère autrement. II est désormais possible de projeter toute image sur tout support; un objet, un écran accroché au poignet et tenu dans la poche, un livre, une architecture, un rêve... Avec le virtuel il n'est même plus besoin d'un support concrêt. L'image incarnée n'a plus de lieu; elle retrouve la mobilité infinie de l'image intérieure; sa dimension impalpable et nomade est décuplée à l'infini et cela va de pair avec l'amplification toujours accrue de sa duplicité. Elle nous conduit de cette manière à la fascination et par la répétition, de la fascination à l'indifférence. Nous nous laissons piéger dans un semblant d'inclusion, dans l'illusion d'une participation sans filtre à un réel ouvert. Nous sommes devenus prisonniers de l'image dirigée; il ne s'agit pas de bannir l'image mais d'être conscient des enjeux qu'elle comporte afin de l'utiliser ou de la déjouer adéquatement. J'ouvre une parenthèse; l'on n'ignore plus que le surgissement des médias en Occident est le fruit d'un monumental projet politique décidé aux origines du christianisme par les philosophes byzantins, désireux d'articuler le pouvoir spirituel au pouvoir temporel, a des fins de pure gestion économique. Un projet qui se précise depuis et qui nous conditionne toujours. Sur ce point, on pourra consulter les importantes recherches publiées par Marie-Josée Mondzain, philosophe, directrice de recherche au CNRS : Icône et image et L'image peut-elle tuer?

Cela me direz-vous concerne-t-il vraiment la gravure ? II ne faut pas oublier que la gravure et l'imprimerie ont été les premiers médias, développés d'ailleurs en Occident - ce qui n'est pas fortuit-même s'ils furent inventés ailleurs; outils de diffusion de démocratisation, mais aussi de contrôle de la pensée; moyens d'une ambiguité déjà certaine dès leurs premières utilisations. Cela concerne directement les domaines de la gravure ainsi que tous les moyens de production et de reproduction de l'image; cela devient la responsabilité des artistes qui utilisent ces moyens; cela permet encore de saisir l'ampleur et la difficulté de l'articulation d'une image artistique à son contenu référentiel et symbolique, ainsi qu'au choix adéquat d'un médium pour la recevoir; un choix qui dépend de ces nombreux aspects; de la double nature conceptuelle de cette image, de sa qualité singulière, de ce que l'on décide ou souhaite lui faire dire. Ce n'est pas un choix qui va dépendre d'une facilité technique ou du simple plaisir que donne l'illusion de contrôle d'un médium. II n'est plus possible de travailler l'image ou de considérer ses moyens de production en ignorant sa non-innocence première. Tous nos gestes même celui de tracer le contour d'une rose sont politiques; ils sont porteurs d'un contenu qui sera lu dans une vision politique; une perspective venant infirmer ou confirmer certaines valeurs culturelles; valeurs que l'art doit secouer ou déplacer, plus souvent sur le mode d'une énonciation plastique que d'une dénonciation directe.

Après ce détour, permettez-moi de revenir à mes quatre images au mur, de les considérer à un autre niveau: Images d'origines semblables, épinglées côte à côte dans le dessein un peu candide d'y déceler des constantes. Je vais vous demander de les imaginer. II ne siérait pas de les présenter en diapositives. Celle-ci est un médium en soi qui trahit l'échelle et la luminosité des autres moyens. Les différences ténues, car il y en a tout de même, dont je souhaite parler seraient entièrement nivelées. Et après tout, cette mise en scène au mur n'est elle-même qu'une pure image. II vous appartiendra de retenir certaines observations, de les vérifier 
ou de les transformer. Imaginez donc sur ce mur un dessin, une photographie, une photogravure et une impression au jet d'encre. Des ombres. Je me suis attardée en premier lieu au dessin; plus chaud croit-on et qui semble toujours si près; près de la main, près du geste, ne laissant soupçonner aucun écart de temps, comme sil venait tout juste d'être configuré. II se livre ainsi dans une contraction temporelle, une illusion d'immédiateté qui me fait adhérer aisément à son rythme, aux finesses de ses qualités, comme à une musique qui serait produite par la voix. Je note qu'il est plein d'incidents, d'événements, de ces petites irrégularités qui lui confèrent une dimension infinie, ouverte à toutes les reprises, les recommencements, les changements; son degré de finitude n'étant jamais que celui que je décide d'arrêter à un point indéterminé de son exécution. Entre l'activité de dessiner et sa résultante, le dessin, il n'y a en apparence que très peu d'écart. Celui-ci prend vie et se construit rapidement, sur les incidents que son processus engendre. Dessiner, c'est découper, opérer des choix dans le vif de l'action. L'effet nous semble toujours direct et tout au plus, inscriton un ralentissement dans son élaboration afin de s'assurer que le geste subséquent sera juste. Le temps de reculer, de reprendre son souffle, de vérifier du regard. Le corps est en action dans une immédiateté totale. C'est ce qui ferait la particularité du dessin. À y repenser, ce que je viens de décrire peut fort bien être attribué à la prise de vue photographique. Là aussi je découpe dans le vif d'un réel, plus vaste bien sûr qu'un rectangle de papier blanc, mais déjà passablement circonscrit par mes propres limites imaginaires et physiques. Je sélectionne, je cadre, jorganise dans un espace toujours rectangulaire, le viseur, ce qui va constituer l'image photographique. Là encore, le corps doit bouger, se déplacer, se plier, s'étendre pour suivre les exigences du regard. Car c'est le regard qui reste le maître, activé dans la pleine découverte. Je prévois le temps et l'ouverture; je contrôle déjà les valeurs finales que je vais donner à l'image. Cette étape devra se répéter à l'impression différemment. II existe donc un premier palier, un premier niveau où se constitue une matrice première; le négatif. Bien que ce choix du négatif définitif est souvent postérieur à la prise de vue. Et je peux décider au départ de procéder intuitivement ou aléatoirement comme pour un dessin, lorsque $j$ 'inscris sur la feuille une première trace sans aucune intention, simplement pour contrer le traumatisme de l'espace vierge et instaurer une contrainte permettant d'amorcer le processus créateur. Si le dessin peut s'élaborer dans un continu de l'activité, mais ce n'est pas un absolu, l'image photographique exige que cette activité se transpose et se transforme de la prise de vue à l'impression. II y a une rupture temporelle inévitable et souvent importante s'inscrivant au cœur de la production d'une photographie. Fréquemment, d'ailleurs, cette attente indéterminée et variable entre les deux étapes, la prise de vue et l'impression, amène des bifurcations, des changements de parcours, des choix autres que ceux qui sont effectués en un premier temps. Ici une autre évidence s'impose; nous sommes forcés de convenir que ces paliers de mûrissement de l'image se retrouvent dans tous les autres moyens de reproduction; dans la gravure, la lithographie, la sérigraphie, même dans l'impression numérique et la vidéographie. Ils peuvent êtres antérieurs ou concomitants à la finalisation de l'œuvre, s'eliminer ou revenir au cours de celle-ci, en permettant de préciser l'image dans un sens souvent inattendu. Les médiums dans leurs manipulations concrètes diffèrent, mais le processus créateur reste sensiblement le même; fait de décisions, de choix, d'attentes nécessaires, de guet de ces événements qui le feront bifurquer. Dans le milieu de l'estampe, j'ai souvent entendu ce lieu commun; la gravure c'est l'accident. Ce qui sous-entend évidemment qu'il faille composer avec cet accident. Ce phénomène n'est pas, je crois, le propre de la gravure. Celle-ci comporte, comme tous les autres moyens de l'art des résistances venant de la non-constance de la matière qui trahissent l'effet prévu ou escompté et qui nous obligent à une bifurcation; il y a des résistances inattendues mais heureuses qui ouvrent sur des voies imprévues; mais il y a aussi celles qui proviennent d'une inadéquation technique; nous savons tous que c'est un véritable champ de bataille. Cet aspect de l'inadéquation technique, retourné positivement, serait celui de la justesse du médium manifeste dans toute œuvre résolue. Là encore, il faut convenir que la maîtrise d'un médium ne se situe pas dans la connaissance de sa manipulation directe mais dans la connaissance de ses possibilités et limites. Cela n'est plus à débattre. Et ce point n'est pas particulièrement contemporain, même si beaucoup d'artistes maintenant travaillent en industrie. On se rappellera, sans insister, les ateliers anciens où les tâches relatives aux exécutions étaient réparties entre des individus spécialisés. II reste que la mise en forme d'une œuvre, peu importe que le moyen soit direct ou non, exige que l'artiste demeure en alerte, prêt à un déplacement, à une dérogation qui lui est suggérée entre autres facteurs, par la matière utilisée et ses résistances.

Revenons de nouveau à mon mur. Chaque manifestation d'une image dans un médium différent la précise autrement. Bizarrement, ce n'est pas le dessin qui ici se fait le plus abstrait; j'entends ici, par abstraction, la possibilité d'une distance mentale et imaginaire accrue, donnée à l'image par le moyen même sans qu'il soit tenu compte de celle déjà implicite dans son statut premier d'image; car 
il y aurait lieu analyser divers niveaux d'abstraction d'une image. Par abstraction, j'entends encore un niveau d'indétermination de l'image qui assure celui qui la regarde d'une certaine latitude; d'une liberté de lecture. Ce n'est pas davantage la photographie. Elle conserve encore trop fortement cette charge culturelle qui fait qu'on la regarde comme un duplicata du réel et cela même si nous n'avons plus de naïveté à ce niveau. II semble que ce soit l'impression numérique qui accentue davantage cette dimension; il s'en dégage un sentiment ambigu d'irréalité; cette image est prélevée du réel, elle le reproduit même en haute-fidélité, mais nous en sentons extrêmement éloignés. Par certains filtres particuliers au médium, elle acquiert une précision figurative qui amplifie celle de la photographie, mais paradoxalement elle se détache davantage du réel; par cette perfection qui permet d'en éliminer tout détail fortuit ou accidentel, toute anecdote, tout incident; par l'immatérialité que le grain numérique confère au grain photographique. Nous sommes dans la dimension d'un lissé surréel. Cette image est à peine crédible. S'il s'agit d'une impression au jet d'encre, l'effet se redouble. Les encres au pigment rappellent le dessin, une sorte d'épure distante, mais l'extrême régularité de leur application par une multitude de petits points égaux éloigne l'image de ce rapprochement plausible. La photogravure par contre se fait très près du dessin. Elle acquiert une qualité qui se situerait entre la photographie et le dessin. Même passablement précise, elle aurait cette supposée chaleur si singulière du dessin, sans doute donnée par la qualité des encres et des pigments et leur dispersion inégale amenée par l'essuyage manuel. Dans ces comparaisons, II ne faut pas pour autant s'imaginer que l'impression numérique est facile et constante. La constitution du fichier numérique est de l'ordre du dessin. L'instrument n'est pas traditionnel, mais sa maîtrise dépend surtout d'un regard éduqué et exercé, et le fait de bien connaître le fonctionnement d'un logiciel est parfaitement insuffisant. Quant à l'impression, elle est tout aussi farcie de dérapages et d'accidents que l'estampe traditionnelle. Si le fichier comme matrice première est une constante, l'impression ne l'est pas. Je dirais même qu'elle comporte autant sinon plus d'aléatoire que l'impression traditionnelle. Selon le calibrage de l'imprimante, la qualité très variable du support, le technicien même, selon parfois d'insondables caprices de la mécanique, il se produit des écarts énormes dans la couleur. Et il faut alors reprendre à tâtons le fichier original, ce qui est un travail monstre. Nous observons encore des lignes rythmiques imprévues qui dépendent de l'ajustement des têtes de l'imprimante. II y a aussi les accidents inscrits dans le fichier qui ont échappé à l'œil nu et se révèlent dans la lecture de l'imprimante. La résultante, malgré cela, regardée rapidement, si on ne compare pas une même image à une autre, semble toujours près de la perfection car l'image est donnée dans un champ exécutoire continu, cette régularité mécanique d'une multitude de points d'encre. L'impression tient vraiment de l'imprimé industriel. Avec l'eau-forte, nous avons une certaine perte de netteté; un grain plus irrégulier provoqué par la morsure inégale de l'acide ou l'irrégularité des molécules d'encre inégalement dispersées, L'inconstance de l'épaisseur d'encre. J'ai longtemps pensé que la gravure était plus adéquate pour des œuvres petites, exigeant une approche plus intime; que la sérigraphie ou l'impression numérique restaient souhaitables pour des images de grandes dimensions; que l'impression numérique était trop parfaite pour de petites images... J'ai du revoir ces certitudes récemment, devant des gravures de Richard Serra; de grandes dimensions, 120 $\mathrm{cm} X \mid 30 \mathrm{~cm}$, montrant des dessins de courbes larges et texturées, lourdement imprégnées d'encre, d'une extrême densité; une épaisseur d'encre noire matérielle, profondément incrustée dans le support rappelant la force de ses sculptures d'acier. Le tout prend un poids que jamais le dessin n'aurait pu donner; encore moins la photographie ou l'impression numérique.

II n'y a donc pas de moyen plus adéquat qu'un autre, plus facile ou plus simple qu'un autre, convenant à tel ou tel type d'image. L'adéquation du médium est à définir à chaque œuvre, à chaque image; selon le contenu dont cette image est porteuse.

Tous les médiums restent viables avec leurs propriétés singulières. Ces propriétés, il faut les regarder comme des filtres venant accentuer ou atténuer une dimension d'inclusion ou d'exclusion toujours inhérente à l'image choisie; une dimension illusoire ou fictive plus ou moins forte définissant le statut de cette image, et le rapport de distance et de liberté que nous entretenons avec elle.

Ce jeu des filtres devrait être regardé comme le ton en musique avec toutes les possibilités de modulations et lidée de calques ou de couches propres au médium numérique, bien que déjà partie du dessin et de la peinture, pourrait être reprise métaphoriquement dans cette notion de filtre; une notion qui va déterminer la distance imaginaire ou physique souhaitable dans une image donnée. Parmi ces filtres; la précision ou l'imprécision, la régularité ou l'irrégularité du grain, de la distance et l'échelle, le près et le lointain et tout autre qualité qui viennent moduler l'intensité et la temporalité de l'image. Tous les moyens de l'art restent ouverts et il ne saurait exister de hiérarchie ou de prédominance d'un moyen sur l'autre. II n'y a de juste que l'adéquation d'un moyen à la résolution d'une l'œuvre. Je m'arrête ici. Je suis toujours devant ce mur qui me laisse malgré tout dans l'irrésolution; hantée par une citation faisant image, tirée de Histoire du cinéma de Godard; elle pointe les abus apportés à 
l'image par ses moyens infinis de reproduction : « II faut dire en silence et avec une seule image ce qui peut être dit en silence et avec une seule image 11.

\section{RÉFERÊNCES}

MONDZAIN, Marie-José. Image, Icône, Économie: Les Sources Byzantines de I'Imaginaire Contemporain. Paris: Seuil, 1996.

MONDZAIN, Marie-José. L'Image peut-elle tuer? Paris: Bayard, 2002.

GODARD, Jean-Luc. Histoire du cinéma. Gaumont, Video $266 \mathrm{~min}, 1998$.

JOCELYNE ALLOUCHERIE: Vit et travaille à Montréal. A enseigné à l'École des Arts Visuels de l'Université Laval, à l'Université du Québec à Montréal, à l'Université Concordia et à l'Université d'Ottawa. À travers des configurations complexes, son oeuvre explore de manière conceptuelle et poétique des notions relatives à l'image, à l'objet et au lieu. A réalisé de nombreuses installations qui associent des éléments relevant de considérations sculpturales, architecturales et photographiques. Sa carrière a été reconnue par nombreux prix et a fait l'objet d'importants textes critiques. http://jocelynealloucherie.com 
